\title{
Dynamic Tensile Behaviour of Strain-Hardening Cement-Based Composites (SHCC)
}

\author{
Iurie Curosu ${ }^{1}$, Viktor Mechtcherine ${ }^{1}$, Daniele Forni ${ }^{2}$, Ezio Cadoni ${ }^{2, *}$ \\ ${ }^{1}$ Institute of Construction Materials, Technische Universität Dresden, Germany \\ ${ }^{2}$ DynaMat Laboratory, University of Applied Sciences of Southern Switzerland (SUPSI), Switzerland
}

\begin{abstract}
The paper presents a part of an experimental campaign consisting of quasi-static and impact tensile experiments on three different types of strain-hardening cement-based composites (SHCC) as well as on their constitutive cementitious matrices [1]. The investigation on different SHCC types was intended for analysing the effect of matrix composition and fibre type on the strain rate sensitivity of the composites and for enabling the formulation of material design recommendations for impact resistant SHCC. The dynamic tests were carried out by means of a Modified Hopkinson Bar (MHB) installed in the DynaMat Laboratory in Lugano, Switzerland, which enabled the characterization of the dynamic material behaviour under direct tensile loading in terms of force-displacement relationships.
\end{abstract}

\section{Introduction}

The vast majority of the civil constructions are made of reinforced concrete (RC). Due to the low tensile strength and pronounced brittleness of concrete, RC structures feature a relatively low resistance against various types of dynamic loading, such as caused by earthquake, impact or blast. The steel reinforcement can only partly compensate these drawbacks, since the distinct cracking, spalling and fragmentation of concrete under severe dynamic loading may render the reinforcement ineffective, especially in supporting columns [2-3]. Moreover, even if the load bearing capacity is not dangerously impaired, the explosive concrete spalling and fragmentation represent a separate risk for people's lives. The performance of existing RC structures under dynamic loading can be enhanced by applying strengthening layers. Normally, their function is to increase load bearing capacity, stability and to ensure a strong confinement of the concrete core. With regard to dynamic loading, the structural elements should also permit considerable inelastic deformations and ensure energy dissipation without losing load bearing capacity. In this sense strain-hardening cement-based composites (SHCC) represent a promising solution [4].

SHCC consist of finely grained cementitious matrices and high-performance polymer micro-fibres usually in volume ratios of up to $2 \%$. The composites exhibit a strain-hardening tensile behaviour associated with a pronounced multiple cracking before failure localization. Owing to their sufficiently high tensile and compressive strength, high ductility and remarkable crack control, SHCC can enhance the load bearing capacity, damage tolerance and durability of structures, both if used in strengthening layers [5-8] or as main material in structural elements [9-10]. The usage of SHCC for strengthening purposes is also motivated by the possibility of applying thin but effective layers on elements of various shapes and with difficult accessibility, e.g. by spraying [8].

However, if not purposefully designed, SHCC may exhibit a negative strain rate sensitivity in terms of strain capacity. The reason for this is that the mechanical properties of SHCC's main constitutive phases, i.e. matrix, fibres and their interfacial bond, exhibit different strain rate sensitivities relative to each other, leading to an alteration of the balance between the crack propagation and crack bridging characteristics when the composite is loaded at rates different from those considered in the material design [11-12]. Thus, counting with a high ductility and energy dissipation of SHCC at high loading rates just based on their tensile performance under quasi-static conditions is not sound.

The results presented in the paper at hand demonstrate the importance of a targeted material design for achieving impact resistant SHCC. A part of a comprehensive experimental series is presented, consisting of quasi-static and impact tension experiments on three different types of SHCC. Two of the composites were made of a normal-strength finely grained cementitious matrix and were reinforced with polyvinylalcohol (PVA) and high-density polyethylene (HDPE) fibres, respectively. The third type consisted of a highstrength finely-grained cementitious matrix and was reinforced with HDPE fibres. The detailed report was published in [1].

\subsection{Tensile testing of SHCC at high strain rates - previous studies}

Corresponding author: ezio.cadoni@supsi.ch 
The MHB used in the current study enabled displacement speeds of up to $6 \mathrm{~m} / \mathrm{s}$, while the initial loading rate was $650 \mathrm{GPa} / \mathrm{s}$. The characteristics of its tensile loading pulse were suitable for achieving failure of 50-mm long ductile SHCC specimens and for yielding an accurate quantitative description of the material behaviour in terms of force-displacement relationships. This was of special importance, because the setups used in previous studies showed obvious limitations in this respect [12-14].

The previously performed experiments involving high-speed hydraulic testing machines failed in describing reliably the response of the specimens due to the strong oscillations caused by the setup ringing [12].

At their turn, the tensile impact experiments involving a split Hopkinson pressure bar yielded a poor signal quality, which was presumably caused by the passage of the compressive wave through the SHCC specimen before being reflected as a tensile loading wave [13]. Furthermore, since the indirect tensile wave was generated by a compressive strike, its length was rather small for ductile SHCC, limiting inconveniently the specimen length. Additionally, the magnitude of the initial strike should be limited to avoid compressive damage of the specimens, which implies a limitation of the strain rate.

Finally, the spall experiments with the Hopkinson bar failed in achieving failure of unnotched ductile SHCC specimens [14]. Also, the reliability of the evaluation method [15] is negatively affected by the ductility of the samples, while recording the load-displacement histories in the specimens is not possible in spall tests.

\section{Materials under investigation}

The investigated SHCC are different combinations of cementitious matrices and polymer micro-fibres. The normal-strength matrix has the index M1 and the highstrength matrix was named M2. Two different types of polymer fibres were included in the study, namely PVA and HDPE, both being normally used in SHCC. The composites were named according to their constitutive cementitious matrices and fibres.

The investigated PVA fibres are high-tenacity Kuralon K-II REC15 fibres produced by Kuraray, Japan [17]. Their diameter is $40 \mu \mathrm{m}$ and the cut length is $12 \mathrm{~mm}$. According to Kuraray, they have a tensile strength of $1600 \mathrm{MPa}$ and a Young's modulus of $40 \mathrm{GPa}$, while the elongation at break is $7 \%$. Because their pronounced hydrophylic nature results in an excessively strong chemical bond with the cement-based matrices, these PVA fibres are coated with an oiling agent during production, which is an effective measure for limiting the bond strength [18-19]. An excessively strong interfacial bond would otherwise lead to a premature fibre rupture during crack formation and, consequently, to a brittle failure of the composite. Nevertheless, the oiling of the PVA fibres surface should be complemented by further adjustments of the matrix composition. The normal-strength matrix M1 was specifically designed for the described 12-mm long PVA fibres. To achieve a sufficient limitation of the interfacial bond strength and to ensure a lower matrix cracking stress compared to the collective crack bridging strength of the fibres, a considerable amount of fly ash was added as cement replacement, see Table 1 . The relatively large fly ash particles may also act as micro-defects and crack initiators, which is favourable for multiple cracking (quasi-ductility). A moderate amount of fine quartz sand was added to ensure a certain Young's modulus. A larger dosage of fine sand would have led to an alteration of the fibre distribution and to an unfavourable increase in matrix's fracture toughness. M1-PVA is a common type of SHCC, which can be found in literature with very similar compositions [16], and it served as reference in the current study.

Table 1. Mixture proportions of the investigated SHCC.

\begin{tabular}{|c|c|c|c|}
\hline SHCC type & M1-PVA & M1-PE & M2-PE \\
\hline Ingredients & {$\left[\mathrm{kg} / \mathrm{m}^{3}\right]$} & {$\left[\mathrm{kg} / \mathrm{m}^{3}\right]$} & {$\left[\mathrm{kg} / \mathrm{m}^{3}\right]$} \\
\hline CEM I 42.5R-HS & 505 & 498 & - \\
\hline $\begin{array}{c}\text { CEM I 52.5 R- } \\
\text { SR3/NA }\end{array}$ & - & - & 1460 \\
\hline Fly ash & 621 & 612 & - \\
\hline Silica fume & - & - & 292 \\
\hline Quartz sand & 536 & 528 & 145 \\
\hline $\begin{array}{c}\text { Viscosity modifying } \\
\text { agent }\end{array}$ & 4.8 & 4.7 & - \\
\hline $\begin{array}{c}\text { Water } \\
\text { Superplasticizer }\end{array}$ & 338 & 333 & 315 \\
\hline $\begin{array}{c}\text { PVA fibre } \\
\text { (2\% vol.) }\end{array}$ & 26 & 25 & 35 \\
\hline $\begin{array}{c}\text { HDPE fibre } \\
\text { (2\% vol.) }\end{array}$ & - & 20 & 20 \\
\hline
\end{tabular}

The HDPE fibres are produced by DSM, the Netherlands, under the product name Dyneema SK62 [20]. They have a tensile strength of $2500 \mathrm{MPa}$ and a Young's modulus of $80 \mathrm{GPa}$. Their cut length is also $12 \mathrm{~mm}$, but the diameter is just $20 \mu \mathrm{m}$, i.e. half the diameter of the PVA fibres. The elongation at break is $3.5 \%$. As opposed to the PVA fibres, the HDPE fibres are highly hydrophobic. Because of this, but also because of their smaller diameter, the matrix developed for these fibres should have an even further reduced grain size, an increased packing density and strength. Therefore, the HDPE fibres are commonly used in combination with high-strength matrices, such as M2 in this work; see Table 1, or similar matrices by other authors [21-22]. As opposed to M1, M2 has a high cement content, a reduced water to binder ratio and, instead of fly ash, silica fume was used as additional binder and filler. The amount of quartz sand was reduced considerably due to the twice smaller diameter of the fibres.

For a straightforward investigation of the fibre type on the strain rate sensitivity of the composites, the normal-strength matrix M1 was also combined with HDPE fibres in M1-PE. The composition of M1 matrix 
was not adapted to the HDPE fibres, which resulted in poor fresh state properties and fibre dispersion, even though the dosage of superplasticizer was increased; see Table 1. This combination was first described in [14]. Despite the mediocre tensile behaviour under quasi-static conditions, in spall experiments this SHCC showed notably high fracture energy.

\subsection{Specimen production}

The same specimen geometry was tested under quasistatic and dynamic loading conditions for enabling a direct comparison of the results at different strain rates. The fresh SHCC materials were initially casted as prismatic beams of dimensions $75 \mathrm{~mm} \mathrm{x} 75 \mathrm{~mm} \mathrm{x}$ $280 \mathrm{~mm}$. The beams were transversally cut in blocks with lengths according to the testing conditions and investigated length of the specimens [1]. The specimens tested quasi-statically had a bigger total length necessary for the $12.5 \mathrm{~mm}$ embedment at both ends (see next section). The specimens were subsequently core drilled from the obtained blocks and the ends were polished for ensuring parallel faces. The diameter of the specimens was $20 \mathrm{~mm}$ as imposed by the MHB.

\section{Testing setups}

\subsection{Setup for quasi-static tension testing}

For the quasi-static tensile tests, the specimens were glued at both ends in steel rings, which were fixed through steel rods to the crossmember and to the force sensor of the machine, respectively; see Fig. 1. A bicomponent glue produced by Evonik Röhm GmbH was used for this purpose. Its tensile strength was insufficient for an end-face gluing of the specimens (that is why rings were used), but its short hardening time allowed testing several specimens per day. The deformations were measured with the help of two linear variable differential transducers (LVDT) attached to the steel rings through a specially built frame. The displacement rate was $0.05 \mathrm{~mm} / \mathrm{s}$.

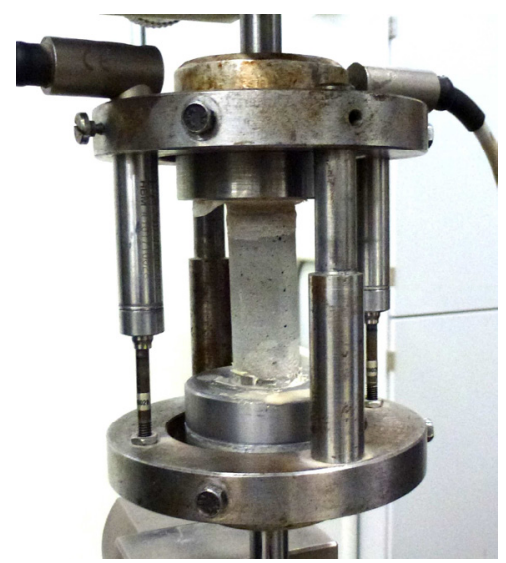

Fig. 1. Quasi-static testing setup with an SHCC specimen.

\subsection{Modified Hopkinson bar setup}

In the MHB the specimens were glued directly to the input and output bars; see Fig. 2. In this case a stronger glue was necessary. A bi-component epoxy resin BASF Concresive 1402 was used, which required at least 8 hours hardening time at room temperature. This allowed only one experiment on SHCC per day. Since it was preferred to test specimens from the same batch for each parameter combination, the testing ages varied between 13 day and 15 days, with 3 specimens tested for each parameter set (i.e. material combination, length, unnotched or notched configuration).

The MHB setup consisted of a 3-m long input bar and of a $6 \mathrm{~m}$ long output bar, both being made of aluminium and having a diameter of $20 \mathrm{~mm}$. A $6 \mathrm{~mm}$ long high-strength steel bar having a diameter of $12 \mathrm{~mm}$ is used as a pre-tension bar for generating the loading pulse; see Fig. 2. The diameter of the pre-tensioned bar was chosen to obtain the same acoustical impedance of the input bar and avoid and spurious reflection at their connection.

During the experiment, the free end of the pretensioned bar is pulled with the help of a hydraulic jack, while the end connected to the input bar is fixed by a blocking device. By pulling the free end of the pretensioned bar, elastic energy is stored in it. Then the rupture of the brittle intermediate piece in the blocking device is followed by a sudden release of the stored elastic energy, generating a tensile wave of trapezoidal shape of $2.4 \mathrm{~ms}$ duration and with a rise-time of about $60 \mu \mathrm{m}$. In the present investigation, the target maximum displacement speed was $6 \mathrm{~mm} / \mathrm{s}$, while the loading rate in the ascending branch of the loading pulse is $650 \mathrm{GPa} / \mathrm{s}$.

The generated pulse propagates along the input bar with a velocity of the elastic wave with its shape remaining constant. When the incident pulse reaches the specimen, part of it is reflected by the specimen due to the different impedances of the specimen material and aluminium, whereas another part passes through the specimen and propagates into the output bar. The relative amplitudes of the incident, reflected and transmitted pulses depend on the mechanical properties of the specimen. Strain gauges glued to the input and output bars of the device are used to measure as a function of time the elastic deformation created on both bars by said incident, reflected and transmitted pulses. The application of the elastic, uniaxial stress wave propagation theory to the Hopkinson bar system [23] allows calculation of the forces and displacements acting on the two faces of the specimen in contact with the input and output bars, respectively. For details regarding the loading characteristics and evaluation protocols see $[1,24]$. 
(2)

(4)

(8)

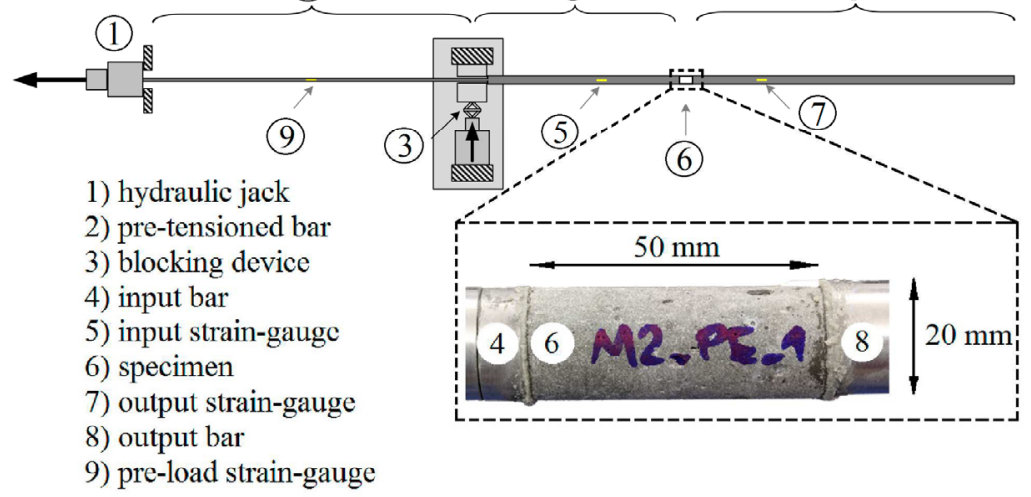

Fig. 2. Modified Hopkinson bar setup with a long SHCC specimen [24].

\section{Results and discussion}

\subsection{Quasi-static tension tests}

Fig. 3 presents the stress-strain curves obtained on SHCC specimens in quasi-static tension tests. The curves are very similar to those presented by the authors in previous works on larger cylindrical specimens $75 \mathrm{~mm}$ in diameter, $250 \mathrm{~mm}$ effective length and $70 \mathrm{~mm}$ gauge length $[14,28]$. The first-crack stress of M1-PVA is in average the highest among the three tested types of SHCC. This is a result of the strong chemical bond between the PVA fibres and the cementitious matrix, which involves the fibres in hindering the propagation of micro-cracks already before steady-state cracking (uncontrolled crack propagation through the entire crosssection) as indicated by the first stress drop in the stressstrain curve. The average first-crack stress of M1-PE is 2.4 MPa, considerably lower in comparison to M1-PVA. The hydrophobicity of the HDPE fibres leads to a weak frictional bond with the normal-strength cementitious matrix M1 meaning that the fibres are activated mainly after crack formation.

Despite the relatively low first-crack stress, M2-PE yielded the highest tensile strength with an average value of $6.5 \mathrm{MPa}$. The other composites reached average tensile strength values of only 3.8 MPa (M1-PVA) and 3.6 $\mathrm{MPa}$ (M1-PE). The fracture surfaces of M2-PE showed partial fibre rupture, indicating on an adequate utilization of the fibre's mechanical properties. At the same time, the fracture surfaces of M1-PVA exhibited a very pronounced fibre rupture, the maximum protruding length of the fibres being less than $2 \mathrm{~mm}$, significantly less than half the fibre length. This was caused not only by the strong interfacial bond, but also by the sliphardening pull-out behaviour of the PVA fibres in the cementitious matrix under low strain rates [14]. In this case the tensile strength of the composite was limited by the tensile strength of the PVA fibres.

In contrast to M1-PVA, the fracture surfaces of M1PE showed complete fibre pull-out, since neither a chemical bond (like in M1-PVA) nor a dense and uniform surrounding matrix (like in M2-PE) enabled a proper fibre anchorage, this explaining the lowest tensile strength. However, the twice smaller diameter of the HDPE fibres ensured a higher number of crack bridging fibres and compensated for the weak fibre-matrix bond. This led to a rather small difference between the tensile strength of M1-PE and M1-PVA.
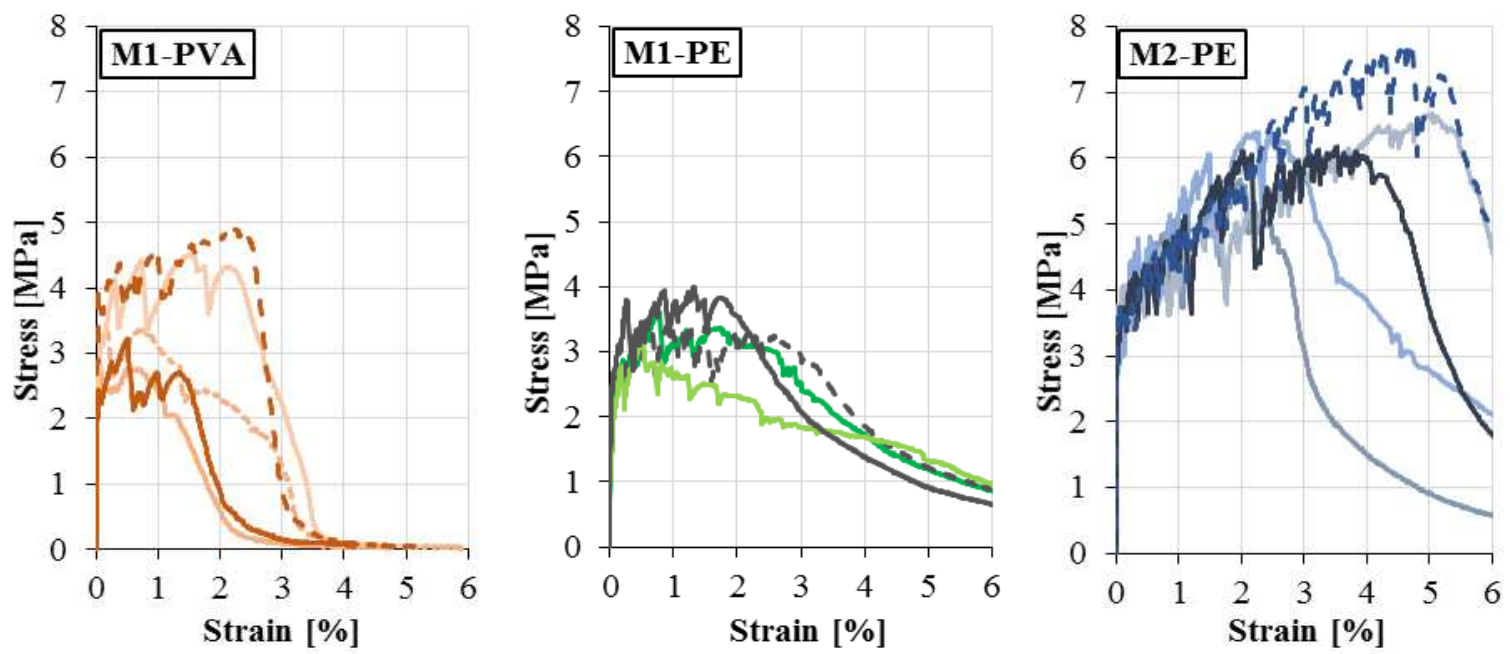

Fig. 3. Stress-strain curves of the SHCC under investigation as obtained in quasi-static tension tests. 
As a result of a pronounced multiple cracking, M2PE reached the highest ultimate strain (before softening) of $3.6 \%$ in average. On one side this was facilitated by the high margin between the first-crack stress and tensile strength (strain-hardening ratio), while on the other it was aided by the defects in the matrix, such as air voids and small distributed clumps of unhydrated silica fume and cement. The latter are caused by the low water-tobinder ratio, by the high content of fines and by the absence of larger hard particles which could break the clumps during the intensive mixing process. The strain capacity of M1-PVA was the lowest compared to the other two SHCCs. An important reason for such a behaviour was the poor multiple cracking due to the excessively strong fibre-matrix bond. An excessively strong interfacial bond does not allow an early enough debonding of the fibre from the matrix (interfacial crack propagation), in this way hindering the formation of a sufficient fibre free-length in the crack, which is necessary for a safe fibre elongation.

The work-to-fracture of the tested SHCC was calculated as the area under the stress-strain curves up to failure localization (softening). The average work-tofracture of M2-PE is $206.5 \mathrm{~kJ} / \mathrm{m}^{3}$, which is approximately four times higher than the fracture energies of the other two SHCC. M1-PVA and M1-PE yielded comparable results of $52.7 \mathrm{~kJ} / \mathrm{m}^{3}$ and $53.5 \mathrm{~kJ} / \mathrm{m}^{3}$, respectively. Based on this, it could be assumed that the high-strength SHCC (M2-PE) is the most appropriate for impact loading conditions. However, as it will be shown in the next section, such a simplified approach is not reliable.

\subsection{Tension experiments at high strain rates}

Fig.4 presents the responses of the specimens in terms of stress-strain curves as recorded in the transmitter bar of the MHB. All the curves show initial force peaks, which are noticeably higher than the forces associated to subsequent deformations and multiple cracking of the specimens. This stronger initial response is a result of the structural inertia caused by the increasing strain rates in the initial loading phase. The comparison of the forces at both specimen ends (not shown here) indicated that no stress equilibrium was reached in the specimens prior to cracking (during the initial loading phase). Stress equilibrium was reached after the initial crack formation only. Because of this, the apparent first-crack stresses were calculated considering the averaged forces at both specimens' ends according to the equations given in [1,24-27]. The dynamic first-crack stress will not be discussed in this article because it needs to be analysed in context with the dynamic tensile experiments on nonreinforced matrices.

After the initial loading phase, the specimens were subjected to relatively constant strain rates; see Fig.4. Under these conditions the structural inertia is negligible and the recorded stress-strain histories yield reliable values for tensile strength and strain capacity. The target displacement rate (flat phase of the input wave) in the performed experiments was $6 \mathrm{~m} / \mathrm{s}$, corresponding to strain rates of $120 \mathrm{~s}^{-1}$. However, the effective strain rates depend not only on the characteristics of the loading pulse, but also on the properties of the tested material. The stress oscillations observed in Fig. 4 are caused by the damage developing in the deforming specimens, crack formations in the first place, but possibly also other phenomena, such as fibre pull-out.

M1-PVA yielded the lowest average tensile strength of just 6.4 MPa, with a dynamic increase factor of DIF = 1.7. Only one M1-PVA specimen showed strainhardening and multiple cracking (curve marked with an arrow); the other two specimens exhibited strainsoftening. M1-PVA showed the lowest increase in tensile strength and a negative influence of high strain rate on the strain capacity, if considering the strainsoftening behaviour of two out of three tested specimens. Nevertheless, the average work-to-fracture increased from $52.7 \mathrm{~kJ} / \mathrm{m}^{3}$ to $90 \mathrm{~kJ} / \mathrm{m}^{3}$.

The change of the failure mode of M1-PVA when subjected to high-speed tensile loading was reported before in $[12,28]$ and confirmed in this investigation.
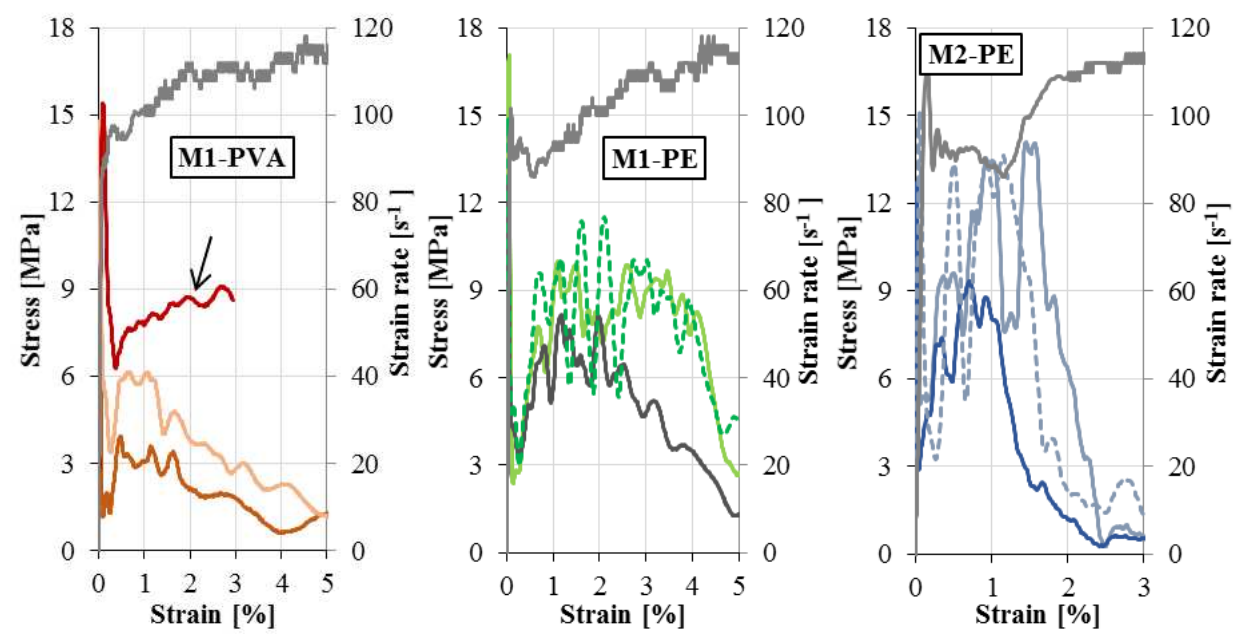

Fig. 4. Stress-strain and strain rate-strain curves from high-speed tension tests on SHCC; note the different strain axes' limits. 
The failed specimens developed few cracks near the fracture zone, while the fracture surfaces showed a complete fibre pull-out. The possible reasons for this were formulated in $[1,14]$.

The average tensile strength of M1-PE is $9.9 \mathrm{MPa}$, with a DIF of 2.8, yielding the highest increase among the tested SHCC. Such an enhancement of the crack bridging capacity indicates a pronounced increase of the interfacial bond strength, which is in good agreement with the single fibre pull-out investigations presented in [14]. The pronounced enhancement of the interfacial bond strength had a beneficial influence on the dynamic strain-capacity of M1-PE as well. The average ultimate strain of M1-PE increased from $1.7 \%$ under quasi-static conditions to $3.3 \%$ under dynamic loading. The optical analysis of the specimens showed a remarkably pronounced multiple cracking. The enhanced tensile behaviour of M1-PE under dynamic loading resulted in a strong increase of the work-to-fracture from $53.5 \mathrm{~kJ} / \mathrm{m}^{3}$ (quasi-static) to $283.6 \mathrm{~kJ} / \mathrm{m}^{3}$ (dynamic).

M2-PE developed the highest average tensile strength of 12.4 MPa, but with a DIF off just 1.9. The average ultimate strain deduced from the stress-strain curves is $1.2 \%$ only, which means a triple reduction in comparison to the corresponding average value of $3.6 \%$ obtained quasi-statically. This can be traced back to considerably less pronounced multiple cracking [1]. The fracture surfaces of M2-PE specimens in the MHB showed significantly more pronounced fibre rupture when compared to those tested in the quasi-static regime. As reported in [14], the increase in fibre-matrix bond strength due to the dynamic loading eclipses the corresponding increase in fibre tensile strength. While the HDPE fibres in the high-strength matrix M2 are optimally utilized under quasi-static conditions, bond strength being appropriate, the excessive increase in bond strength at high strain rates leads to premature fibre rupture during crack formation and, consequently, to reduced quasi-ductility. Due to this, M2-PE showed a clear reduction of work-to-fracture, from $206.5 \mathrm{~kJ} / \mathrm{m}^{3}$ to $115.3 \mathrm{~kJ} / \mathrm{m}^{3}$.

\section{Conclusions}

The strain rate sensitivity of SHCC is strongly influenced by their fibre type and matrix composition and their tensile behaviour under quasi-static loading is not representative for that under high strain rates. The relatively low dynamic enhancement of the fibre-matrix bond in M1-PVA at high strain rates leads to a dramatic reduction in quasi-ductility. At the same time, the frictional interactions between the HDPE fibres with the normal-strength and high-strength cementitious matrices seem to have a pronounced dynamic enhancement. In the high-strength SHCC this leads to a more pronounced fibre rupture and to a reduction in quasi-ductility, while in the normal-strength SHCC made with HDPE fibres this has a beneficial effect, ensuring a notable increase in the tensile strength, strain capacity and fracture energy.

\section{References}

1. I. Curosu, V. Mechtcherine, D. Forni, E. Cadoni. Cem. Concr. Res. 102 16-28 (2017)

2. H. Aoude, F.P. Dagenais, R.P. Burrell, M. Saatcioglu. Int. J. Impact Eng. 80 185-202 (2015)

3. T.R. Brewer, J.E. Crawford, K.B. Morrill, P.J. Vonk, K.A. Horiuchi. Proc. 4th Int. Conf. Protective Structures (ICPS4) (2016)

4. V. Li. J. Adv. Concr. Technol. 1(3) 215-230 (2003)

5. M. Kunieda, K. Rokugo. J. Adv. Conc. Technol. 4(1) 19-33 (2006)

6. M. Hussein, M. Kunieda, H. Nakamura. Cem. Concr. Comp. 34 1061-1066 (2012)

7. M. Kunieda, N. Ueda, H. Nakamura, T. Tamakoshi. FraMCoS-8 (2013)

8. V. Mechtcherine, Constr. Build. Mater. 41 365-373 (2013)

9. Y.Y. Kim, B.Y. Lee, J.W. Bang, B.C. Han, L. Feo, C.G. Cho. Composites : Part B $56512-519$ (2014)

10. T. Kanda, Y. Takaine, S. Tamoe, M. Takahashi, Y. Yamamoto, K. Kawano, M. Kunieda, T. Mizobuchi. SHCC3 409-414 (2014)

11. E.H. Yang, V.C. Li. Constr. Build. Mater. 52 96-104 (2014)

12. V. Mechtcherine, F.D.A. Silva, M. Butler, D. Zhu, B. Mobasher, S.L. Gao, E. Mäder. J. Adv. Concr. Technol. 9(1) 51-62 (2011)

13. I. Curosu, A. Ashraf, V. Mechtcherine. SHCC3 121128 (2014)

14. I. Curosu, V. Mechtcherine, O. Million. Cem. Concr. Res. 82 23-35 (2016)

15. H. Schuler, C. Mayrhofer, K. THoma. Int. J. Impact Eng. 32 1635-1650 (2006)

16. E.H. Yang, V. Li. Cem. Concr. Res. 421066 (2012)

17. Kuralon Data Sheet, http://www.kuraray.co.jp/kii/english/

18. C. Redon, V.C. Li, C. Wu, H. Hoshiro, S. Tadashi, A. Ogawa. J. Mater. Civil. Eng. 13 399-406 (2001)

19. V.C. li, C. Wu, S. Wang, A. Ogawa, T. Saito. ACI Mater. J. 99(5) 463-472 (2002)

20. Dyneema Fact Sheet, Eurofibers (2010)

21. R. Ranade, V.C. Li, W.F. Heard. Cem. Concr. Res. 68 94-104 (2015)

22. A. Kamal, M. Kunieda, N. Ueda, H. Nakamura. Cem. Concr. Comp. 30(10) 863-871 (2008)

23. H. Kolsky. Proc. Phys. Soc. B 62 676-700 (1949)

24. E. Cadoni, A. Meda, G. Plizzari. Mater. Struct. 42(9) 1283-1294 (2009)

25. E. Cadoni, D. Forni, EPJ-ST 225(2), 253-264 (2016)

26. A. Caverzan, E. Cadoni, M. di Prisco, Int J Imp Engg 45(0), 28 - 38 (2012)

27. E. Cadoni, Rock Mec. Rock Eng 43, 667-676 (2010)

28. V. Mechtcherine, O. Million, M. Butler, K. Thoma. Cem. Concr. Comp. 33 1-11 (2011) 